\title{
Shamanskii method for solving parameterized fuzzy nonlinear equations
}

\author{
Ibrahim Mohammed Sulaiman $^{a}$, Mustafa Mamat ${ }^{a, ~ *}$, Puspa Liza Ghazali ${ }^{b}$ \\ ${ }^{a}$ Faculty of Informatics and Computing, Universiti Sultan Zainal Abidin, Kuala Terengganu, Malaysia \\ ${ }^{b}$ Faculty of Business and Managemnet Sciences, Universiti Sultan Zainal Abidin, Kuala Terengganu, Malaysia \\ sulaimanib@unisza.edu.my,must@unisza.edu.my,puspa@unisza.edu.my
}

\section{ARTICLE INFO}

Article history:

Received: 24 July 2019

Accepted: 12 April 2020

Available Online: 10 December 2020

Keywords:

Shamanskii method

Fuzzy nonlinear equations

Parameterized fuzzy equations

Numerical experiments

AMS Classification 2010:

$34 G 20,93 C 10$

\begin{abstract}
One of the most significant problems in fuzzy set theory is solving fuzzy nonlinear equations. Numerous researches have been done on numerical methods for solving these problems, but numerical investigation indicates that most of the methods are computationally expensive due to computing and storage of Jacobian or approximate Jacobian at every iteration. This paper presents the Shamanskii algorithm, a variant of Newton method for solving nonlinear equation with fuzzy variables. The algorithm begins with Newton's step at first iteration, followed by several Chord steps thereby reducing the high cost of Jacobian or approximate Jacobian evaluation during the iteration process. The fuzzy coefficients of the nonlinear systems are parameterized before applying the proposed algorithm to obtain their solutions. Preliminary results of some benchmark problems and comparisons with existing methods show that the proposed method is promising.
\end{abstract}

\section{Introduction}

Nonlinear systems have played important role in solving problems arising from numerous areas of application which include, engineering, medicine, social sciences. However, the parameters of these systems are often in the form of fuzzy numbers (FN). Thus, the result rely on solutions of fuzzy equation [1]. The notion of fuzzy numbers (FN) was first presented by Zadeh [2]. Some standard analytical procedures of Buckley and Qu [3], [4], are only suitable for the linear and quadratic case of nonlinear equations. For any equations of the form;

(1). $a y^{3}+b y^{2}+c y-d=e$

(2). $a e^{y}+b=d$

where $y, a, b, c, d$, and $e$ are fuzzy numbers. Because the standard analytical techniques are unable to solve fuzzy nonlinear equations (FNE), numerical methods to obtain the solution of the problems are important. At this point, two propensity are seemingly visible. Firstly, we witness augmenting classical and existing numerical approaches, which are often well established, by applying them to obtain the solution of FNE. The aim is to provide the existing methods with insight and new functionalities of the subsequent system. Secondly, this is the focus of recent researches, by looking at the trend of real treatment of previous problems, we can substantially reformulate thereby coming up with new solution methods in a fully innovative fashion for solving fuzzy nonlinear equations.

Most numerical methods considered requires the computation and storage of either Jacobian or approximate Jacobian at every iteration. For example, after parameterizing the fuzzy quantities, Newton's approach [1] was employed to solve the corresponding FNE. Newton's method is known to convergence rapidly when starting with an initial guess that is chosen near the solution point [5]. The disadvantage of this scheme is computing and inverting of the Jacobian at each step of the iteration. Likewise, Quasi Newton's technique [8] was applied to obtain the root of FNE. This technique requires the storage of approximate Jacobian at every iteration. Also, the Chord Newton's method [6] was proposed for computing the root of dual FNE. However, the Chord technique also needs the computation of Jacobian either at first iteration or after few iterations. Recently, Regula Falsi method [7] was applied to solve FNE. This technique is the simplest and the most reliable one among bracketing approaches for finding the root of nonlinear equations. However, it has a poor rate of convergence due to lack of derivative information. 
To overcome some of these drawbacks, Shamanskii method [8] was applied to obtain the solution of FNE. This scheme starts by computing the first iterates using the Newton step, and subsequently, computes the Chord steps in other iterations. The reason is to reduce the cost of computing the Jacobian at all iteration, just like in other numerical methods. Also, for nonsingular Jacobian and the initial points chosen near the solution point, then, the proposed Shamanskii scheme will converge q-superlinearly [9].

The remaining part of this article will contain the following. In the next section, we present preliminary discussions on fuzzy operations. Section 3 describes the derivation process of Shamanskii scheme for solution of nonlinear equation. In section 4 , we present the proposed Shamanskii method for solution of FNE. Results of some examples are presented in section 5. Finally, concluded in section 6.

\section{Preliminaries}

Some useful results are defined as below.

Definition 2.1. A fuzzy number can be defined as a set $h: \mathbb{R} \rightarrow I=[0,1]$ satisfying the below criteria [10]

\section{1. $h$ is upper semi-continuous}

2. $h(x)=0$ outside some interval $[c, d]$

3. there exist $a, b \in \mathbb{R}$ such that $c \leq a \leq b \leq d$ and $3.1 h(x)$ is increasing monotonically on $[c, a]$

$3.2 h(x)$ is decreasing monotonically on $[b, d]$

$3.3 h(x)=1, a \leq x \leq b$

In the rest of the paper, we shall use the symbol $E$ to denote the set of all fuzzy numbers whose parametric form is defined in [10, 11].

Definition 2.2. $h: \mathbb{R} \rightarrow I=[0,1]$ in parametric form refer to the pair $(\underline{h}, \bar{h})$ of $\underline{h}(\propto), \bar{h}(\propto), 0 \leq \propto \leq 1$ satisfying [10, 12],

(1) $\underline{h}(\propto)$ is an increasing left continuous function that is monotonically bounded.

(2) $\bar{h}(\propto)$ is a decreasing right continuous function that is monotonically bounded.

(3) $\underline{h}(\propto) \leq \bar{h}(\propto), 0 \leq \propto \leq 1$.

Definition 2.3. A classical Fuzzy number $h$ refers to the Triangular number $h=(a, b, c)$ given as follows

$$
h(x)= \begin{cases}\frac{(x-a)}{(c-a)}, & a \leq x \leq c, \\ \frac{(x-b)}{(c-b)}, & c \leq x \leq b,\end{cases}
$$

with $h(x)$ known as the membership function and $c \neq$ $a, c \neq b$ [1], [9]. This function can be written in its parameterized form as follows

$$
\begin{aligned}
& \bar{h}(\propto)=b+(c-b) \propto, \\
& \underline{h}(\propto)=a+(c-a) \propto .
\end{aligned}
$$

Assume $T F(\mathbb{R})$ denotes the set of all trapezoidal fuzzy number. The operation of scalar multiplication and addition can be extended to fuzzy number using the extension principle given below [1].

Let $h=(\underline{h}, \bar{h}), g=(\underline{g}, \bar{g})$, with $k>0$, the addition $(u+v)$ and multiplication by scalar $k$ are defined as

$$
\begin{gathered}
(\overline{g+h})(\propto)=\bar{g}(\propto)+\bar{h}(\propto), \\
(\underline{g+h})(\propto)=\underline{g}(\propto)+\underline{h}(\propto), \\
\hline(\overline{k g})(\propto)=k \bar{g}(\propto), \\
(\underline{k g})(\propto)=k \underline{g}(\propto) .
\end{gathered}
$$

For more references on properties of fuzzy set, please refer to $[13,14]$.

\section{Shamanskii method for nonlinear equations}

Consider the following nonlinear equation

$$
F(x)=0
$$

Systems of the form (1) are often solved by Newton's type methods. Starting with an initial guess $x_{c}$, we obtain the new iterate say $x_{+}$, by computing $F\left(x_{c}\right)$. Then check the stopping criteria either to terminate or proceed with the iteration. If one is to proceed, then one must compute and factored the Jacobian matrix $F^{\prime}\left(x_{c}\right)$ to enable one to compute the step as the solution of $F^{\prime}\left(x_{c}\right) s=-F\left(x_{c}\right)$.

Newton's method updates the new iterate as

$$
x_{+}=x_{c}-F^{\prime}\left(x_{c}\right)^{-1} F\left(x_{c}\right)
$$

which will converge quadratically to $x^{*}$ if the starting guess $x_{c}$ is suitably chosen close to $x^{*}$ and $F^{\prime}\left(x^{*}\right)$ is nonsingular [15]. However, computing and storage of the Jacobian at every iteration are very expensive and time-consuming. A variation of this method that almost entirely overcame the drawback of this method is the Chord method which only computes $F^{\prime}\left(x_{0}\right)$. The iterates are defined as follows.

$$
x_{+}=x_{c}-F^{\prime}\left(x_{0}\right)^{-1} F\left(x_{c}\right)
$$

In the case of a finite-dimensional problem, the Jacobian matrix would be factored only once and thereby reducing the computational cost of each iteration. This degrades the convergence rate to linear $[15,16]$. In fact, if the $x_{0}$ is chosen close to $x^{*}$ and $F^{\prime}\left(x^{*}\right)$ is nonsingular, then for some constant $K_{c}>0$, we have

$$
\left\|x_{n+1}-x^{*}\right\| \leq K_{c}\left\|x_{0}-x^{*}\right\|\left\|x_{n}-x^{*}\right\|
$$


This, however, improves the convergence rate as the initial guess increase. Motivated by the idea, a method due originally to Shamanski [8] that lies between Chord-Newton and Newton's methods was proposed and has been analyzed in [16]. The Shamanskii method moves through an intermediate sequence says $\left\{W_{k, p}\right\}_{p=1}^{m}$, that is, one Newton's iterate and subsequent iterates are computed using the Chord steps [9], [17]. The convergence analysis of the considered Shamanskii scheme has been established.

Theorem 1 [18]. Suppose $F: D \subset R^{n} \rightarrow R^{n}$ conform to hypotheses $H 1(2), H 2$, and $H 3$. Then, the solution point $x^{*}$ is referred to the attraction point of the Shamanskii process defined in (7) with at least $m+1$ order of convergence.

\section{Shamanskii method (SM) for solving fuzzy nonlinear equations}

Consider the following:

$$
F(x)=c
$$

Starting with an initial approximation $x_{0}$, the Shamanskii scheme uses the multiple pseudo-Newton approaches as described below.

$$
\begin{gathered}
x_{k+\frac{1}{2}}=x_{k}-F^{\prime}\left(x_{k}\right)^{-1} F\left(x_{k}\right) \\
x_{k+1}=x_{k+\frac{1}{2}}-F^{\prime}\left(x_{k}\right)^{-1} F\left(x_{k+\frac{1}{2}}\right)
\end{gathered}
$$

Parameterizing the fuzzy equation (5), we have;

$$
\left\{\begin{array}{l}
\underline{F}(\underline{x}, \bar{x}, r)=\underline{c}(r), \quad 0 \leq r \leq 1 \\
\bar{F}(\underline{x}, \bar{x}, r)=\bar{c}(r) .
\end{array}\right.
$$

To obtain the solution of the above equation, we generate sequence $\left\{x_{k}\right\}$ that converge to the solution points $x^{*}$ via the Algorithm 1 below.

\section{Algorithm 1: Shamanskii algorithm for FNE}

Step 1: Given the FNE in parameterized form

Step 2: Obtain $F\left(x_{k}\right)$ and $F\left(x_{k+\frac{1}{2}}\right)$

Step 3: Compute $x_{k+\frac{1}{2}}=x_{k}-F^{\prime}\left(x_{k}\right)^{-1} F\left(x_{k}\right)$

Step 4: Set $x_{k+1}=x_{k+\frac{1}{2}}-F^{\prime}\left(x_{k}\right)^{-1} F\left(x_{k+\frac{1}{2}}\right)$

Step 5: Update $x_{k+1}=$

$$
x_{k}-F^{\prime}\left(x_{k}\right)^{-1}\left[F\left(x_{k}\right)+F\left(x_{k}-F^{\prime}\left(x_{k}\right)^{-1} F\left(x_{k}\right)\right)\right]
$$

Step 6: Continue the process with the next $k$ until tolerance conditions $\epsilon \leq 10^{-4}$ are achieved.

Note that for $m=1$, the method reduces to the Newton's method, while for $m=\infty$, we have chord method [3].

\section{Remark}

The sequence $\left\{\underline{x}_{k}, \bar{x}_{k}\right\}_{k=0}^{\infty}$ converges to $(\underline{\lambda}, \bar{\lambda}$,$) iff \forall r \in$ $[0,1]$

and

$$
\lim _{k \rightarrow \infty}(\underline{x}(r))=\underline{\lambda}(r)
$$

$$
\lim _{k \rightarrow \infty}(\bar{x}(r))=\bar{\lambda}(r) \text {. }
$$

\section{Numerical results}

This section presents results to illustrates the Shamanskii method based on number of iterations for FNE. Results of three examples show the efficiency of the proposed method. All algorithm is coded on MATLAB 7.0 (R2013) subroutine programming. The CPU processor used was Intel ${ }^{\circledR}$ corei5-2410M. The problems considered are from [1], [19], [20].

Example 1. Consider the FNE

$$
(4,6,8) x^{2}+(2,3,4) x-(8,12,16)=(5,6,7)
$$

Without loss of generality (WLOG), let $x$ be positive, we define the parameterized equations as follows.

$$
\begin{aligned}
& (4+2 r) \underline{x}^{2}(r)+(2+r) \underline{x}(r)-(3+3 r) \\
& (8-2 r) \bar{x}^{2}(r)+(4-r) \bar{x}(r)-(9-3 r)
\end{aligned}
$$

For the initial point, we compute for $r=0$ and $r=1$. For $r=0$, we have

$$
\begin{aligned}
& 4 \underline{x}^{2}(0)+2 \underline{x}(0)=3 \\
& 8 \bar{x}^{2}(0)+4 \bar{x}(0)=9
\end{aligned}
$$

and for $r=1$

$$
\begin{aligned}
& 6 \underline{x}^{2}(1)+3 \underline{x}(1)=6 \\
& 6 \bar{x}^{2}(1)+3 \bar{x}(1)=6
\end{aligned}
$$

Thus, $\underline{x}(0)=0.6514, \bar{x}(0)=0.8397$, and $\underline{x}(1)=$ $\bar{x}(1)=0.7808$, which are near to the exact solution. Therefore, we set as $x_{0}=(0.6,0.8,0.9)$. The desired results obtained by the proposed method and other existing methods used for comparison are given in Table 1 . With an error less than $10^{-4}$, we obtain.

Table 1. Performance Comparison for Problem 1

\begin{tabular}{cc}
\hline Methods & $\begin{array}{c}\text { Number of } \\
\text { Iterations }\end{array}$ \\
\hline Newton's Method & 2 \\
Chord Method & 4 \\
Shamanskii Method & 3 \\
\hline
\end{tabular}

See Figure 1 for details description of the solution. 


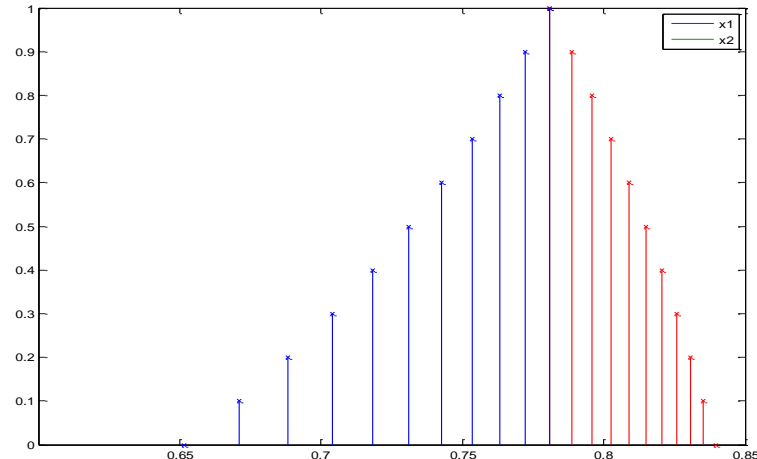

Figure 1. Solution of Shamanskii method for Problem 1

Example 2. Consider the FNE

$$
(3,3,4,5) x^{2}+(1,2,3)=(1,1,2,3)
$$

WLOG, let $x$ be positive, we define the parameterized equations as follows

$$
\begin{aligned}
& (3+r) \underline{x}^{2}(r)+(1+r) \underline{x}(r)=(1+r) \\
& (5-r) \bar{x}^{2}(r)+(3-r) \bar{x}(r)=(3-r)
\end{aligned}
$$

For the starting point, we solve the above equations for $r=0$, which gives

$$
\begin{aligned}
& 3 \underline{x}^{2}(0)+\underline{x}(0)-1=0 \\
& 5 \bar{x}^{2}(0)+\bar{x}(0)-3=0
\end{aligned}
$$

and for $r=1$

$$
\begin{aligned}
& 4 \underline{x}^{2}(1)+2 \underline{x}(1)-2=0 \\
& 4 \bar{x}^{2}(1)+2 \bar{x}(1)-2=0
\end{aligned}
$$

Thus, we have $\underline{x}(0)=0.4343, \bar{x}(0)=0.5307$, and $\underline{x}(1)=\bar{x}(1)=0.5000$, which is near the solution. Hence, to demonstrate the efficiency of the method, we defined a new initial point $x_{0}=(0.4,0.5,0.6)$. The desired results obtained by the proposed method and other existing methods used for comparison are given in Table 2. With an error less than $10^{-4}$, we obtain.

Table 2. Performance Comparison for Problem 2

\begin{tabular}{cc}
\hline Methods & $\begin{array}{c}\text { Number of } \\
\text { Iterations }\end{array}$ \\
\hline Newton's Method & 2 \\
Chord Method & 4 \\
Shamanskii Method & 2 \\
\hline
\end{tabular}

See Figure 2 for details of the solution.

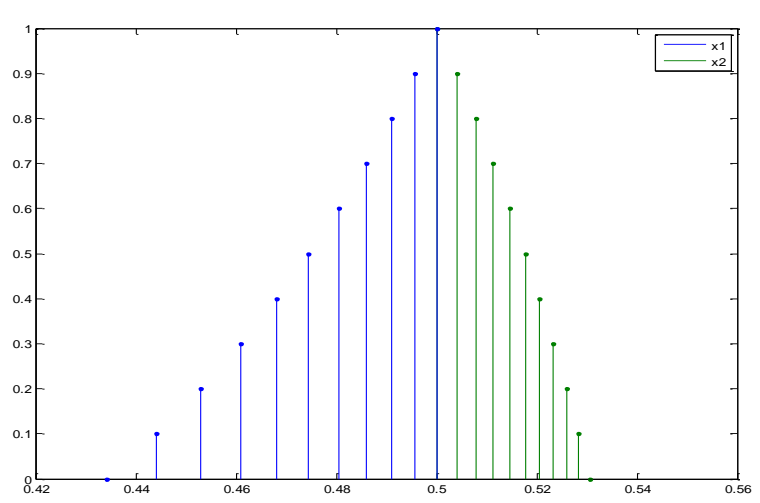

Figure 2. Solution of Shamanskii method for Problem 2.

\section{Example 3.}

Suppose an establishment wishes to invest around one million dollars $(K=1,0.2,0.2)$ at interest rate $R$ so that after one calendar year they would be likely to withdraw approximately 250,000 dollars $\left(S_{1}=\right.$ $0.25,0.02,0.05)$ and after the second year, the amount that is left will accrue to about 900,000 dollars $\quad\left(S_{2}=0.9,0.3,0.3\right)$. Find the rate $R$ so that $A$ can enough to cover about $S_{1}$ and $S_{2}$. The rate $R$ will be a FN with its support in $[0,1]$.

\section{Solution.}

At the end of the first year, the amount in the corporation's account would be

$$
K+K R
$$

Also, after withdrawing the amount $S_{1}$, the amount at the beginning of the second year would be

$$
K-S_{1}+A R
$$

At the end of the second year, the accrued amount is

$$
\left[\left(K-S_{1}\right)+K R\right]+\left[\left(K-S_{1}\right)+K R\right] R
$$

which can be rewritten as

$$
K R^{2}+B R+D
$$

where $B=2 K-S_{1}$ and $D=K-S_{1}$. Thus,

$$
K R^{2}+K R+D=S_{2}
$$

via substitution, we get

$$
\begin{aligned}
& (1,0.2,0.2) R^{2}+(1.75,0.45,0.45) R \\
& +(0.75,0.25,0.25)=(0.9,0.3,0.3)
\end{aligned}
$$

Suppose $R$ is positive, without loss of generality, the above equations are transformed into its parametric form as follows

$$
\begin{gathered}
(0.8+0.2 r) \underline{R}^{2}(r)+(1.3+0.45 r) \underline{R}(r) \\
-(0.1+0.05 r)=0 \\
(1.2-0.2 r) \bar{R}^{2}(r)+(2.2-0.45 r) \bar{R}(r) \\
-(0.2-0.05 r)=0
\end{gathered}
$$

For the starting point, we compute for $r=0$ and $r=1$. For $r=0$

$$
\left\{\begin{array}{l}
0.8 \underline{R}^{2}(0)+1.3 \underline{R}(0)-0.1=0 . \\
1.2 \bar{R}^{2}(0)+2.2 \bar{R}(0)-0.2=0 .
\end{array}\right.
$$


and for $r=1$

$$
\left\{\begin{array}{l}
\underline{R}^{2}(1)+1.75 \underline{R}(1)-0.15=0 \\
\bar{R}^{2}(1)+1.75 \bar{R}(1)-0.15=0
\end{array}\right.
$$

Thus, we have $\underline{R}(0)=0.07359, \bar{R}(0)=0.08680$, and $\underline{R}(1)=\bar{R}(1)=0.08188$. Suppose $R$ is negative, we have $\underline{R}(0)=-1.69859, \quad \bar{R}(0)=-1.92013$. By definition, $\underline{R}(r) \leq \bar{R}(r), \forall r \in[0,1]$. This has shown that negative root of the solution does not exist. For the initial guess, we consider the fuzzy number.

$$
\begin{gathered}
R=(\underline{R}(0), \underline{R}(1) \bar{R}(0)) \\
=(0.07359,0.08188,0.08680)
\end{gathered}
$$

Thus, the starting point is $x_{0}=(0.07359,0.08680)$. The desired results obtained by the proposed method and other Newton-type methods are given in Table 3. With an error less than $10^{-4}$, we obtain.

Table 3. Performance Comparison for Problem 3

\begin{tabular}{cc}
\hline Methods & $\begin{array}{c}\text { Number of } \\
\text { Iterations }\end{array}$ \\
\hline Newton's Method & 3 \\
Chord Method & 4 \\
Shamanskii Method & 3 \\
\hline
\end{tabular}

For detailed exact solution of problem 3, please refer to Table 4 and Figure 3.

Table 4. Solution of Problem 3 for $\left(\underline{x}_{n}(r), \bar{x}_{n}(r)\right)$

\begin{tabular}{ccc}
\hline$r$ & $\underline{x}_{n}(r)$ & $\underline{x}_{n}(r)$ \\
\hline 0 & 0.0735904299 & 0.0867995482 \\
0.1 & 0.0746678539 & 0.0863997233 \\
0.2 & 0.0756758682 & 0.0859829574 \\
0.3 & 0.0766209975 & 0.0857378114 \\
0.4 & 0.0775089705 & 0.0850940961 \\
0.5 & 0.0783448379 & 0.0846194916 \\
0.6 & 0.0791330704 & 0.0841229037 \\
0.7 & 0.0798776402 & 0.0836027651 \\
0.8 & 0.0805820880 & 0.0830573558 \\
0.9 & 0.0812495809 & 0.0824847832 \\
1.0 & 0.0818829605 & 0.0818829605 \\
\hline
\end{tabular}

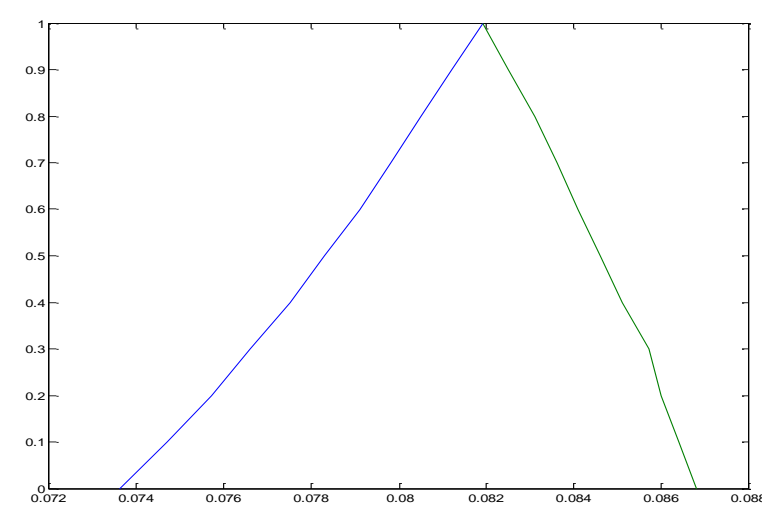

Figure 3. Solution of Shamanskii method for Problem 3.

\section{Conclusion and recommendations}

Recently, numerous studies have been done on computationally efficient numerical algorithms for the solution of FNE. Most of these methods are Newtontype methods that require the computation and storage of Jacobian or approximate Jacobian matrix at each iteration which are usually challenging and timeconsuming. This paper observes the behavior of existing numerical algorithms for solving different fuzzy nonlinear problems and further, proposed the Shamanskii approach to overcome these drawbacks. The Shamanskii algorithm starts by computing Newton's step, followed by several Chord steps. The technique was able to save a lot of the computational burdens of the Jacobian matrix at every iteration. Preliminary results presented show that the proposed method is very competitive when compared to the classical Newton and Chord methods with superlinear convergence rate. This implies that the proposed method can be used as an alternative for solution of fuzzy nonlinear equations.

Recent studies focus on application of conjugate gradient method to real-life situation [21]. This approach can also be extended to solve fuzzy nonlinear equations.

\section{Acknowledgments}

The authors would like to extend their appreciations to all anonymous reviewers for their valuable suggestions and comments.

\section{References}

[1] Abbasbandy, S., \& Asady, B. (2004). Newton Method for solving fuzzy nonlinear equations. Applied Mathematics and Computation, 159, $349-$ 356.

[2] Zadeh, L.A. (1965). Fuzzy sets. Information and Control 8, 338-353.

[3] Buckley J.J., \& Qu Y. (1991). Solving fuzzy equations: a new solution concept" Fuzzy Set and Systems, 39, 291- 301.

[4] Buckley J.J., \& Qu Y. (1990). Solving linear and quadratic fuzzy equations. Fuzzy Sets and Systems $38,43-59$.

[5] Chong, E. K. P., \& Zak, S. H. (2013). An introduction to optimization, Wiley series in discrete mathematics and optimization.

[6] Waziri M.Y., \& Moyi A. (2016). An alternative approach for solving dual fuzzy nonlinear equations. International Journal of Fuzzy Systems, 18, 103 107.

[7] Sulaiman, I. M, Mamat, M, Waziri, M. Y, Fadhilah, A, \& Kamfa, U. K. (2016). Regula Falsi Method for Solving Fuzzy Nonlinear Equation. Far East Journal of Math Sci 100(6), 873-884.

[8] Shamanskii, V.E. (1967). "A modification of Newton's method" Ukrain. Mat. Zh. 19, 133-138.

[9] Kelley C. T. (1995). Iterative Methods for Linear and Nonlinear Equations. SIAM, Philadelphia. 
[10] Dubois, D., \& Prade, H. (1980). Fuzzy Sets and Systems. Theory and Application, Academic Press, New York, NY, USA.

[11] Goetschel, R., \& Voxman, J.W. (1986). Elementary fuzzy calculus," Fuzzy set and Systems, 18, 31-43.

[12] Zimmermann H. J. (1991). Fuzzy Set Theory and its Applications. Third ed., Kluwer Academic, Norwell, MA.

[13] Hulya, G. C. (2018). On the exact and the approximate solutions of second-order fuzzy initial value problems with constant coefficients. Malaya Journal of Matematik, 6(1), 61-68.

[14] Hulya, G. C. (2019). Comparisons of the exact and the approximate solutions of second-order fuzzy linear boundary value problems. Miskolc Mathematical Note, 20(2), 823-837.

[15] Kelley C. T. (1986). A Shamanskii-Like Acceleration Scheme for Nonlinear Equations at Singular Roots. Mathematics of Computation, 47, 609-623.

[16] Traub, J. F. (1964). Iterative Methods for the Solution of Equations. Prentice Hall, Englewood Cliffs, NJ.

[17] Sulaiman, I..M. (2018). New iterative methods for solving fuzzy and dual fuzzy nonlinear equations. $\mathrm{PhD}$ Thesis. Universiti Sultan Zainal Abidin, Malaysia

[18] Kchouk B, \& Dussault J. (2013). The ChebyshevShamanskii Method for Solving Systems of Nonlinear Equations. J Optim Theory Appl 157. 148-167.

[19] Amirah, R., Lazim, M., \& Mamat, M. (2010). Broyden's method for solving Fuzzy nonlinear equations. Advances in fuzzy system, Article ID 763270, 6 pages.

[20] Otadi, M., \& Mosleh, M. (2011). Solution of fuzzy equations by adomian decomposition Method. Soft Computes. 15: 187-192.

[21] Sulaiman, I. M. Yakubu, A. U. \& Mamat, M. (2020). Application of Spectral Conjugate Gradient
Methods for Solving Unconstrained Optimization Problems. An International Journal of Optimization and Control: Theories \& Applications. (Accepted Manuscript).

Ibrahim Sulaiman Mohammed is currently a post-doctoral researcher at Faculty of informatics and computing, Universiti Sultan Zainal Abidin (UniSZA), Malaysia from 2019 till date. He obtained his PhD from UniSZA in 2018 specializing in the field of fuzzy systems. His research interest includes Numerical research, Fuzzy nonlinear systems, unconstrained optimization.

\section{http://orcid.org/0000-0001-5246-6636}

Mustafa Mamat is currently a Professor of Computational and Applied Mathematics at Universiti Sultan Zainal Abidin (UniSZA), Malaysia since 2013. He obtained his PhD from UMT in 2007 specialization in optimization field. To date, he has successfully supervised more than 70 postgraduate students and published more than 260 research papers in various international journals and conferences. His research interest includes unconstrained optimization such as conjugate gradient methods and chaotic systems. Currently, he is the Editor in Chieffor Malaysian Journal of Computing and Applied Mathematics (a UniSZA journal in applied science) and an editor for Indonesian Journal of Science and Technology.

https://orcid.org/0000-0002-4802-3733

Puspa Liza Ghazali is currently an Associate Professor of Business and Management at Universiti Sultan Zainal Abidin (UniSZA), Malaysia since 2018. She obtained her PhD from Universiti Malaysia Terengganu (UMT) in 2013 specialization in Financial Mathematics. Her research interest includes Financial Mathematics, Islamic Insurance, Insurance, Mathematical Science, Statistical Modelling and optimization. Currently, she is the one of the Editorial Board in Journal of Management Theory and Practice (a UniSZA journal in Management).

https://orcid.org/0000-0002-4039-1865

An International Journal of Optimization and Control: Theories \& Applications (http://ijocta.balikesir.edu.tr)

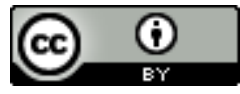

This work is licensed under a Creative Commons Attribution 4.0 International License. The authors retain ownership of the copyright for their article, but they allow anyone to download, reuse, reprint, modify, distribute, and/or copy articles in IJOCTA, so long as the original authors and source are credited. To see the complete license contents, please visit http://creativecommons.org/licenses/by/4.0/. 